\title{
Homology modeling and molecular dynamics studies of Wilms' tumor gene 1 frameshift mutations in exon 7
}

\author{
SHAOYAN HU ${ }^{1}$, YING WANG ${ }^{1}$, SHUIYAN WU ${ }^{1}$, MINGYING ZHANG ${ }^{1}$, JIAN PAN ${ }^{1}$, HONGJIE SHEN ${ }^{2}$, \\ XIAOFEI QI ${ }^{2}$, JIANNONG CEN ${ }^{2}$, ZIXING CHEN ${ }^{2}$, BAIRONG SHEN ${ }^{3}$ and RUIHUA CHEN ${ }^{4}$ \\ ${ }^{1}$ Department of Hematology and Oncology, The Children's Hospital of Soochow University, Suzhou, Jiangsu 215003; \\ ${ }^{2}$ Jiangsu Institute of Hematology, The First Affiliated Hospital of Soochow University, Suzhou, Jiangsu; \\ ${ }^{3}$ Center for Systems Biology, Soochow University, Suzhou, Jiangsu; ${ }^{4}$ Institute of Clinical Immunology, \\ The First Affiliated Hospital of Soochow University, Suzhou, Jiangsu 215006, P.R. China
}

Received May 22, 2013; Accepted July 03, 2013

DOI: $10.3892 /$ br.2013.149

\begin{abstract}
As a transcription factor, the Wilms' tumor 1 (WT1) gene plays an important role in leukemogenesis. The impact of WT1 gene mutations has been reported in acute myeloid leukemia (AML). However, the number of available studies on the spatial configuration changes following WT1 mutation is limited. In this study, we sequenced the mutation in exon 7 of the WT1 gene in 60 children with newly diagnosed AML and the spatial configuration of WT1 with frameshift mutations in exon 7 was evaluated using the software for homology modeling and optimization of molecular dynamics. Three cases with frameshift mutations in exon 7 were identified (3/60; mutation rate, $5 \%$ ). One case had a mutation that had been previously described, whereas the remaining two mutations were first described in our study. Of the three cases, one case presented with antecedent myelodysplastic syndrome (MDS) and the remaining two cases exhibited primary resistance to induction chemotherapy. The spatial configuration analysis demonstrated that the three mutations affected the spatial structure of exon 7 and even affected exon 8 compared to its wild-type. This study demonstrated that the frameshift mutation in exon 7 of the WT1 gene is a poor prognostic factor for children with AML, partly through the spatial configuration changes following frameshift mutations of WT1, which highlights the structure-based function analysis and may facilitate
\end{abstract}

Correspondence to: Professor Shaoyan Hu, Department of Hematology and Oncology, The Children's Hospital of Soochow University, 303 Jingde Road, Suzhou, Jiangsu 215003, P.R. China

E-mail: hsy139@126.com; shaoyanhu67@126.com

Dr Ruihua Chen, Institute of Clinical Immunology, The First Affiliated Hospital of Soochow University, Suzhou, Jiangsu 215006, P.R. China

E-mail: chenrh189@189.cn

Key words: Wilms' tumor gene 1, frameshift mutation, acute myeloid leukemia, children, resistance to chemotherapy, myelodysplastic syndrome the elucidation of the pathogenesis underlying WT1 gene mutations.

\section{Introduction}

The Wilms' tumor 1 (WT1) gene has been extensively used in monitoring minimal residual disease (MRD) in acute myeloid leukemia (AML) as a panleukemic marker, due to its overexpression (1-3), particularly in cases lacking known fusion genes (4). The WT1 gene, which is considered to be an oncogene, is mainly involved in promoting stem cell proliferation and hampering cell differentiation in AML $(5,6)$. However, the number of studies investigating WT1 mutations has been on the increase, although their prognostic significance in predicting the outcome in AML has been controversial (7-11). WT1 mutations are encountered in $~ 10 \%$ of AML patients and the resulting defect affects the interaction of WT1 with other transcription factors, which may contribute to leukemogenesis and resistance to chemotherapy (7). The hotspots of WT1 mutations are mainly located in exon $7(85.41-87.06 \%)(9,10)$, with frameshift mutations leading to the formation of a premature stop codon and a truncated protein lacking the C-terminal zinc fingers. Mutations in exon $9(7.06-8.33 \%)(9,10)$ are rare and predominantly of the missense type, which interrupt DNA binding capacity by affecting the amino acid residues directly involved in DNA binding or essential to the structure of the zinc finger motif (12).

In this study, we screened the rate of WT1 gene mutations in exon 7 and analyzed their effect on pediatric AML. Furthermore, we used the software [ExPASy Translate Tool (http://www.expasy.ch/tools/dna.html)] for homology modeling and optimization of molecular dynamics to evaluate the spatial configuration of WT1 with frameshift mutations in exon 7.

Homology modeling, also referred to as comparative or knowledge-based modeling, develops a three-dimensional model from a protein sequence based on the structures of homologous proteins. Evolutionarily related proteins have similar sequences and naturally occurring homologous proteins have similar structures. It has been demonstrated that the three-dimensional protein structure is evolutionarily more conserved than would 
Table I. Clinical and genetic characteristics of the 60 children with newly diagnostic acute myeloid leukemia.

\begin{tabular}{lccccc}
\hline FAB subtype & Cases & Gender $(\%$ female $)$ & Median age (years) & Median WBC $\left(\mathrm{x} 10^{\%} / 1\right)$ & Karyotype (\% normal) \\
\hline M1 & 2 & 100 & 4 & 19.9 & 50 \\
M2 & 12 & 56 & 6 & 8.7 & 33 \\
M3 & 21 & 53 & 8 & 11.7 & 10 \\
M4 & 13 & 22 & 8.5 & 31.2 & 25 \\
M5 & 12 & 55 & 11 & 38.6 & 38 \\
\hline
\end{tabular}

FAB, French-American-British classification; WBC, white blood cell.

be expected due to sequence conservation (13). By utilizing this method, we demonstrated that WT1 frameshift mutations in exon 7 affected the spatial configuration of WT1.

\section{Materials and methods}

Patients and samples. Cryopreserved bone marrow samples collected at diagnosis from 60 patients with newly diagnosed AML were provided by the Children's Hospital of Soochow University. The diagnosis and classification of AML were based on morphological, cytogenetic and immunophenotypic criteria according to the WHO classification. Children with AML were treated according to the protocol for Chinese AML children, as determined by the Subspecialty Group of Hematology, Society of Pediatrics, Chinese Medical Association (14). More detailed information is provided in Table I.

The study was approved by the ethics committee of the hospital and informed consent was provided by the parents or the legal guardians of the patients. The procedures were approved by the hospital's Institutional Review Board.

Polymerase chain reaction $(P C R)$. For mutation analysis of the exon 7 of the WT1 gene, PCR amplification was performed with the use of specific primers (15): 7F: 5'-CTCCAG TGCTCACTCTCCCTC-3'; 7R: 5'-CCTTAGCAGTGTGAG AGCCTG-3'.

The following PCR conditions were used: $2 \mathrm{~min}$ at $50^{\circ} \mathrm{C}$, $10 \mathrm{~min}$ at $95^{\circ} \mathrm{C}, 35$ cycles for $10 \mathrm{sec}$ at $95^{\circ} \mathrm{C}$ and $30 \mathrm{sec}$ at $60^{\circ} \mathrm{C}$, with a final extension step for $30 \mathrm{sec}$ at $72^{\circ} \mathrm{C}$. The wild-type amplicon comprised 309 base pairs. The purified PCR products were directly sequenced from the two strands using the described primers and analyzed on the Applied Biosystems 3730 Genetic Analyzer (Applied Biosystems, Carlsbad, CA, USA).

Statistical analysis. The sequence data were analyzed using Chromas software version 2.31. According to the structure of the Wilms' tumor suppressor protein zinc finger domain bound to DNA (16), homology modeling and optimization of molecular dynamics was performed with the ExPASy Translate Tool to investigate the spatial configuration of the WT1 gene with frameshift mutations in exon 7.

\section{Results}

Study population. A total of 60 newly diagnosed AML samples collected from The Children's Hospital of Soochow
University, were screened for WT1 gene mutations. The patient characteristics are provided in Table I.

Mutation analysis of the WT1 exon 7. We analyzed the samples for mutations in the exon 7 of the WT1 gene. Of the 60 cases of patients with AML, only three cases harboured a frameshift mutation, which accounts for 5\%. The three cases are described in detail below.

Case 1 had antecedent myelodysplastic syndrome (MDS). Case 1, a 3-year-old female was diagnosed as AML-M1 with a white blood cell (WBC) count of $19.9 \times 10^{9} / 1$ and an abnormal karyotype 46,XX,t(2;11)(q31;p15),del(12)(p12) (15). The patient had a history of MDS for 6 months prior to the diagnosis of AML-M1. Complete remission (CR) was achieved after the first induction therapy. The patient has been on consolidation therapy since CR. The c.[1319delG] was detected, which caused a frameshift mutation compared to the wild-type sequence (Table II). Alanine (Ala) coded by GCC was replaced by proline (Pro) coded by CCC, since $1319 \mathrm{G}$ was deleted and the $1320 \mathrm{C}$ behind $\mathrm{G}$ moved forward to form a new codon with $1317 \mathrm{C}$ and $1318 \mathrm{C}$. Accordingly, all the amino acids behind Ala were changed due to the recombined codons. This mutation was previously reported by Gaidzik et al (15), although not in association with patient characteristics (16). The spatial configuration alterations due to the mutation compared to the wild-type may be visualized based on homology modeling and optimization of molecular dynamics (Fig. 1A and B).

Cases 2 and 3 exhibited primary resistance to chemotherapy. Case 2, a 2-year-old male, was diagnosed as AML-M3 with a WBC count of $53.3 \times 10^{9} / 1$ and a normal karyotype $(46, \mathrm{XY})$. PML-RAR $\alpha$ was negative. CR was achieved after 4 cycles of chemotherapy and the patient relapsed 6 months later. The patient had a mutation in c.[1342delA; 1349-1350insA; 1353delG; 1355-1356insG; 1392delG; 1400-1401insT; 1405delT];c.[1415-1416insC];c.[1431delG];c.[1433-1434insA] (Table II), which had not been reported before.

Case 3, a 6-year-old female, was diagnosed as AML-M2a with a WBC count of $43.6 \times 10^{9} / 1$ and a complex karyotype $46, \mathrm{XX}, \mathrm{t}(8 ; 12 ; 21) / 45, \mathrm{idm},-\mathrm{X}(1,8)$. No positive fusion genes were detected. The ratio of blast cells in the bone marrow was $22 \%$ following administration of daunorubicin, cytarabine and etoposide as induction therapy for 7 days, and even increased to $57 \%$ after a prolonged 3 days of cytarabine. The patient had a mutation in c.[1345delT;1349-1350insA; 1353delG; 
Table II. Sequence of exon 7 of the WT1 gene (wild-type and cases with frameshift mutations).

\begin{tabular}{ll}
\hline Exon 7 & \multicolumn{1}{c}{ Sequence } \\
\hline Wild-type & 1295-GAT GTG CGA CGT GTG CCT GGA GTA GCC CCG ACT CTT GTA CGG TCG GCA \\
TCT GAG ACC AGT GAG AAA CGC CCC TTC ATG TGT GCT TAC CCA \\
GGC TGC AAT AAG AGA TAT TTT AAG CTG TCC CAC TTA CAG ATG \\
CAC AGC AGG AAG CAC ACT G-1445 \\
1295-GAT GTG CGG CGT GTG CCT GGA GTA (del G)CC CCG ACT CTT GTA CGG TCG \\
GCA TCT GAG ACC AGT GAG AAA CGC CCC TTC ATG TGT GCT TAC \\
CCA GGC TGC AAT AAG AGA TAT TTT AAG CTG TCC CAC TTA CAG \\
ATG CAC AGC AGG AAG CAC ACT G-1445 \\
1295-GAT GTG CGA CGT GTG CCT GGA GTA GCC CCG ACT CTT GTA CGG TCG GC(del A) \\
TCT GAG A(ins A)CC A (del G)T G(ins G)AG AAA CGC CCC TTC \\
ATG TGT GCT TAC CCA GGC T(del G)C AAT AAG AGA T(ins T)AT \\
TT(del T) AAG CTG T(ins C) CC CAC TTA CAG AT(del G) CA (ins A)C \\
AGC AGG AAG CAC ACT G -1445 \\
1295-GAT GTG CGG CGT GTG CCT GGA GTA GCC CCG ACT CTT GTA CGG CCG GCT \\
TC(del T) GAG A(ins A)CC A(del G)T G(ins G)AG AAA CGC CCC TTC ATG \\
TGT GCT TAC CCA GGC TGC A(del A)T AAG AGA TAT TTT AAG CTG TCC \\
CAC TTA CAG ATG CAC AGC AGG AAG CAC ACT G-1445
\end{tabular}

del, deletion; ins, insertion; WT1, Wilms' tumor 1 gene. Bold lettering highlights the position and type of mutation.

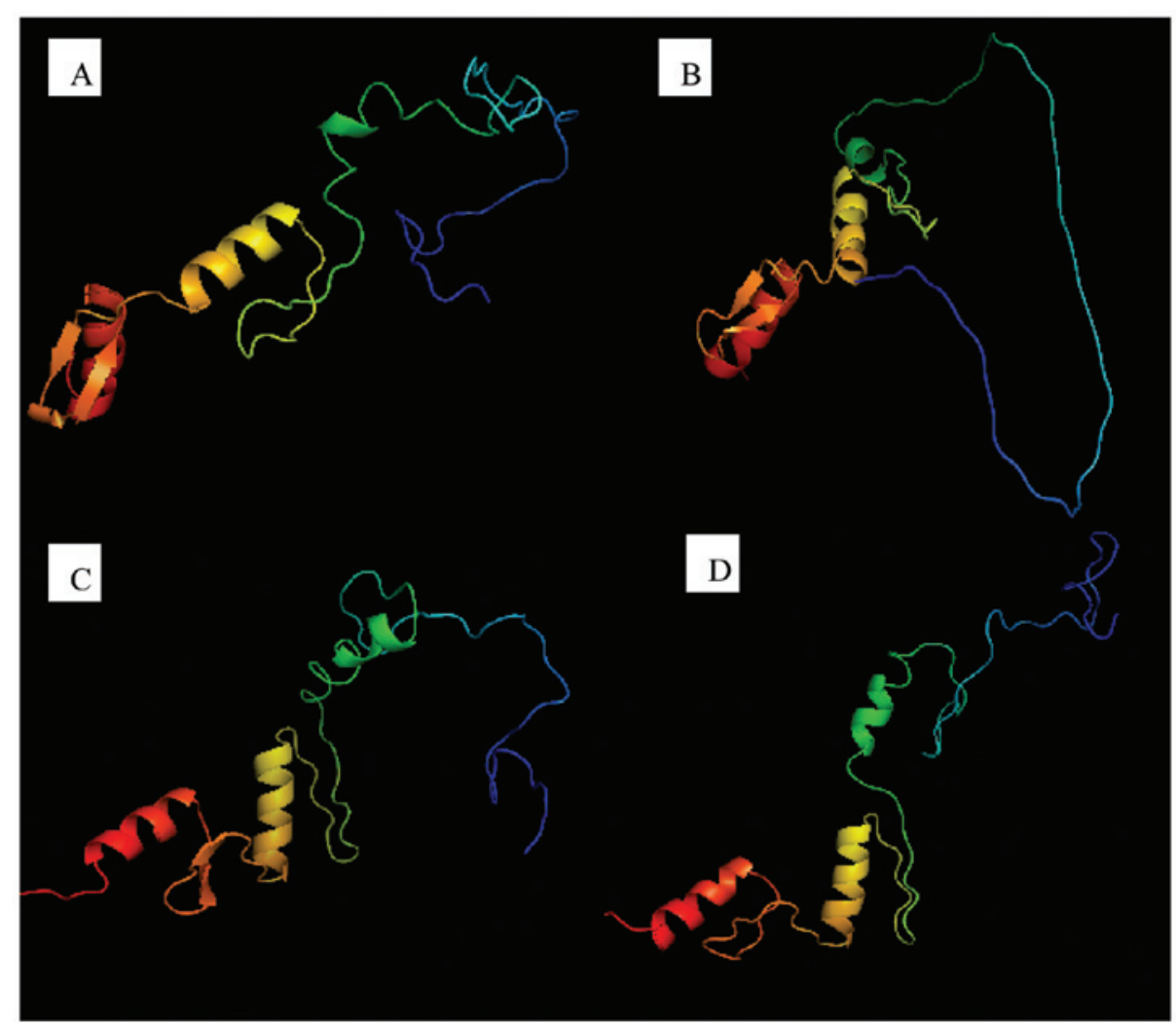

Figure 1. Spatial configuration of exon 7 demonstrated by homology modeling and optimization of molecular dynamics. (A) Wild-type; (B) case 1; (C) case 2; (D) case 3. Blue color, $\mathrm{N}$-terminal coding regulatory regions of other transcription factors; green color, exon 7; orange color, exon 8; red color, exon 9 . Compared to the wild-type, the three cases exhibit distinct alterations in their spatial configuration.

1355-1356insG; 1392delA], which was first reported by our group (Table II).

The mutations in these two cases affected the spatial configuration of exon 7 (Fig. 1C and D), although to a lesser extent compared to case 1 (Fig. 1B).

\section{Discussion}

The WT1 gene is located on $11 \mathrm{p} 13$, encoding a protein of 429 amino acids (17). The protein is constituted of the C-terminal of the DNA-binding region and the N-terminal 
Table III. Mutation panel of AML patients.

\begin{tabular}{rrrllllllll}
\hline ID & Gender & Age & FAB & NPM1 & FLT3 $^{\text {a }}$ & C-kit & DNMT3A 882AA & WT1E7 & WT1E9 & CEBPA \\
\hline 1 & F & 12 & M3v & P & N & N & N & N & N & N \\
2 & M & 8 & M2 & N & N & N & N & N & N & P \\
3 & M & 1 & M4Eo & N & N & N & N & N & N & N \\
4 & F & 3 & M4 & N & P & N & N & N & N & N \\
5 & F & 6 & M2-relapse & N & N & N & N & N & N & N \\
6 & F & 9 & AML & N & P & N & N & N & N & N \\
7 & M & 11 & M3 & N & N & N & N & N & N & N \\
8 & F & 1 & M4Eo & N & N & P & N & N & N & N \\
9 & M & 13 & M2a & N & P & N & N & N & N & N \\
10 & F & 3 & M5b & N & N & N & N & N & N & N \\
11 & F & 3 & M5 & N & N & N & N & N & N & N \\
12 & F & 2 & M5 & N & N & N & N & N & N & N \\
13 & M & 8 & M4 & N & P & N & N & N & N & N \\
14 & M & 9 & M2 & N & N & N & N & N & N & P \\
15 & F & 11 & M5 & N & P & N & N & N & N & N \\
16 & F & 8 & M2 & N & N & P & N & N & N & N \\
\hline
\end{tabular}

aEither FLT3-ITD or FLT3-TKD. AML, acute myeloid leukemia; F, female; M, male; FAB, French-American-British classification; NPM1, nucleophosmin; DNMT3A 882AA, DNA (Cytosine-5-)-methyltransferase 3 $\alpha$ at amino acid postion R882; WT1, Wilms' tumor 1 gene; E7, exon 7; E9, exon 9; CEBPA, CCAT/enhancer binding protein- $\alpha$ double mutation; $N$, negative; P, positive. Bold lettering highlights mutation.

of the transcriptional regulatory region. A previous study by King-Underwood et al (1) was the first to report WT1 gene mutations in AML and their potential effect on AML. Since then, WT1 mutations have been sporadically reported until recently, when the results on WT1 mutations from cohort studies $(8-11,15)$ have begun to attract attention again. However, the effect of WT1 gene mutations on the prognosis of AML varies widely among different groups, even leading to opposite conclusions (8-11). This inconsistency may be attributed to differences in the populations investigated, such as age (children vs. adults), karyotype (normal vs. abnormal), combination with other mutations such as FLT3-ITD, or different ethnicities (17). The mutation rate in exon 7 in our study was 5\% (3/60), which was lower compared to that reported by previous studies on pediatric AML $(9,12)$. This may be due to our limited patient sample or due to the fact that M3 was included in this cohort. Our data demonstrated that the three cases with frameshift mutations had a short term survival which indicated that frameshift mutations may be related to their initial characterics (antecedent MDS or resistance to chemotherapy). Hollink et al (10) reported similar results, according to which WT1 gene mutations conferred an independent poor prognostic significance. By contrast, Ho et al (9) expanded the population to 842 patients and observed that WT1 mutations alone are of no independent prognostic significance in predicting the outcome in pediatric AML. From the data of that study it was indicated that the FLT3-ITD status affected the evaluation of WT1 mutations. We investigated FLT3-ITD, FLT3-TKD, NPM1, CEBPA, C-kit, $D N M T 3 A 882 A A$ and $W T 1$ exon 7 and 9 mutation in 16 cases of AML and did not observe WT1 mutation overlapping with any other mutations (Table III).
Gene expression regulated by transcriptional factors is one of the important regulatory mechanisms in the proliferation and differentiation of hematopoietic cells (18). The WT1 gene is a transcription factor which is crucial in the early differentiation of hematopoietic cells. WT1 may inhibit blood-related gene transcription, such as (Bcl-2, c-Myc and CSF-1) and is closely associated with hematological disorders $(19,20)$. Stoll et al (16) demonstrated that exon 7 (zinc finger 1) played an important role in enhancing the WT1 binding activity with its target DNA in a non-specific manner. Zinc finger structure is a supersecondary structure that is able to regulate transcription by specifically combining with nucleic acid binding sites or by forming connections between the zinc finger proteins. In our study, three cases with WT1 frameshift mutations in exon 7 were demonstrated to exhibit spatial configuration alterations, which may disturb the interaction with other transcription factors, conferring transformation of MDS into AML or leukemia cell resistance to chemotherapy. Further investigations of the effect of WT1 exon 7 mutations on the mechanism of AML are required, with the use of bioinformatics technology.

\section{Acknowledgements}

This study was supported by $81170513,81100371,81170468$ from the National Natural Science Foundation of China, BK2009127 from the Natural Science Foundation of Jiangsu Province, H200921 from the Department of Public Health of Jiangsu Province, 333 Project of Jiangsu Province. The authors would like to thank Professor Chien-Shing Chen, Loma Linda University Medical Center, for his critical review of the manuscript. 


\section{References}

1. King-Underwood L, Renshaw J and Pritchard-Jones K: Mutations in the Wilms' tumor gene WT1 in leukemias. Blood 87: 2171-2179, 1996.

2. Inoue $\mathrm{K}$, Ogawa $\mathrm{H}$, Yamagami $\mathrm{T}$, et al: Long-term follow-up of minimal residual disease in leukemia patients by monitoring WT1 (Wilms tumor gene) expression levels. Blood 88 : 2267-2278, 1996.

3. Inoue K, Sugiyama H, Ogawa H, et al: WT1 as a new prognostic factor and a new marker for the detection of minimal residual disease in acute leukemia. Blood 84: 3071-3079, 1994.

4. Inoue K, Ogawa H, Sonoda Y, et al: Aberrant overexpression of the Wilms tumor gene (WT1) in human leukemia. Blood 89: 1405-1412, 1997.

5. Yang L, Han Y, Suarez Saiz F and Minden MD: A tumor suppressor and oncogene: the WT1 story. Leukemia 21: 868-876, 2007.

6. Ariyaratana S and Loeb DM: The role of the Wilms tumour gene (WT1) in normal and malignant haematopoiesis. Expert Rev Mol Med 9: 1-17, 2007.

7. King-Underwood L and Pritchard-Jones K: Wilms' tumor (WT1) gene mutations occur mainly in acute myeloid leukemia and may confer drug resistance. Blood 91: 2961-2968, 1998.

8. Hou HA, Huang TC, Lin LI, et al: WT1 mutation in 470 adult patients with acute myeloid leukemia: stability during disease evolution and implication of its incorporation into a survival scoring system. Blood 115: 5222-5231, 2010.

9. Ho PA, Zeng R, Alonzo TA, et al: Prevalence and prognostic implications of WT1 mutations in pediatric acute myeloid leukemia (AML): a report from the Children's Oncology Group. Blood 116: 702-710, 2010

10. Hollink IH, van den Heuvel-Eibrink MM,Zimmermann M, et al: Clinical relevance of Wilms tumor 1 gene mutations in childhood acute myeloid leukemia. Blood 113: 5951-5960, 2009.

11. Becker H, Marcucci G, Maharry K, et al: Mutations of the Wilms tumor 1 gene (WT1) in older patients with primary cytogenetically normal acute myeloid leukemia: a Cancer and Leukemia Group B study. Blood 116: 788-792, 2010.
12. Little $\mathrm{M}$ and Wells $\mathrm{C}$ : A clinical overview of WT1 gene mutations. Hum Mutat 9: 209-225, 1997.

13. Kaczanowski S and Zielenkiewicz P: Why similar protein sequences encode similar three-dimensional structures? Theor Chem Acc 125: 543-550, 2010.

14. Subspecialty Group of Hematology Diseases; Society of Pediatrics; Chinese Medical Association; and Editorial Board of Chinese Journal of Pediatrics: Suggestion of diagnosis and treatment of acute myelocytic leukemia in childhood. Zhonghua Er Ke Za Zhi 44: 877-878, 2006 (In Chinese).

15. Gaidzik VI, Schlenk RF, Moschny S, et al; German-Austrian AML Study Group: Prognostic impact of WT1 mutations in cytogenetically normal acute myeloid leukemia: a study of the German-Austrian AML Study Group. Blood 113: 4505-4511, 2009.

16. Stoll R, Lee BM, Debler EW, et al: Structure of the Wilms tumor suppressor protein zinc finger domain bound to DNA. J Mol Biol 372: 1227-1245, 2007.

17. Ho PA, Kuhn J, Gerbing RB, et al: WT1 synonymous single nucleotide polymorphism rs16754 correlates with higher mRNA expression and predicts significantly improved outcome in favorable-risk pediatric acute myeloid leukemia: a report from the Children's Oncology Group. J Clin Oncol 29: 704-711, 2011

18. Maurer U, Weidmann E, Karakas T, et al: Wilms tumor gene (wt1) mRNA is equally expressed in blast cells from acute myeloid leukemia and normal $\mathrm{CD} 34^{+}$progenitors. Blood 90: 4230-4232, 1997.

19. Hewitt SM, Hamada S, McDonnell TJ, Rauscher FJ III and Saunders GF: Regulation of the proto-oncogenes bcl-2 and c-myc by the Wilms' tumor suppressor gene WT1. Cancer Res 55: 5386-5389, 1995.

20. Rossetti S, Van Unen L, Touw IP, et al: Myeloid maturation block by AML1-MTG16 is associated with Csf1r epigenetic downregulation. Oncogene 24: 5325-5332, 2005. 